told me that four months ago he had fallen against a hook on a wall which had caught the lower part of his left eye. On the lower and inner part of the globe was a raised red patch close to the fornix. He had no pain, and his sight in that eye was excellent, but he had all the signs of left superior oblique paralysis which dated from the accident. There was neither any history nor any sign of injury about the upper and inner part of his orbit, but the site of the trochlea was distinctly tender to pressure.

I presume that the blow pressing the eye forcibly upwards and outwards put such a strain on the pulley that it tore.

As the diplopia did not seem to inconvenience him much except when he was reading, I advised him when looking down to tufn his head downwards and to the right, i.e., turning his head in the same direction as the affected muscle, if it acted, would turn his eye. $\mathrm{He}$ seemed to be quite satisfied with this as he could then read comfortably. I told him, if it made him tired, to wear a shade over one or the other eye.

Now, if a patient were not so easily satisfied, it is an interesting point to consider what should be done for him. It would be possible to detach a strip of periosteum from below and make a new pulley by attaching it to the adjoining periosteum, but it might not be strong enough. Prof. Morison, whom I consulted about this problem, suggested adding a little strip of fascia lata. I should be glad to hear of any other suggestions for the purpose, but I am afraid thàt, at any rate in my hands, the result would not be satisfactory to me, although some benefit might be derived.

Since writing the above I have seen, at the Eye Infirmary, another case of trochlear injury. A boy had been kicked by a horse on the right superciliary ridge; little damage resulted except for the complete detachment of his right trochlea, and the consequent inaction of his superior oblique.

\title{
MOBILE CYST OF THE IRIS
}

\author{
BY \\ Edgar Stevenson, \\ CAPTAIN R.A.M.C.
}

THE following account of a curious eye condition is put forward in the hope that it may of interest, though I am fully aware that a mere description of such a case is generally unconvincing. Unfortunately, however, owing to the patient's age and state of health, it is impossible to present her at any scientific meeting away from Liverpool. 
A few days ago my friend, Dr. Heaney of this city, asked me to see an old lady who, he said, was able at will to pour a brown fluid from the posterior chamber of her right eye into the anterior chamber, and then let it flow back again. This sounded rather like a conjuring trick, but when I came to examine the patient, I found that his description was almost literally accurate.

The patient, aged 67 years, is rather frail looking, but with no organic disease, and her statement, corroborated by her daughter, is that this "appearance" first took place quite suddenly, and without any pain about ten years ago. It has persisted ever since, and has been a sort of a joke in the family, but latterly it seemed to be getting larger; and she thinks it is beginning to affect her sight. It also annoys her. She is conscious. when it takes place, and when it happens, as it often does, in a tram-car, she thinks people will notice it. About four years ago she had an operation for gall stones. "It" then disappeared altogether for about six months, and returned quite suddenly one morning while she was having breakfast.

When the eyes are examined in strong daylight, the only abnormality noticed is that in the iris of the right eye (both irides being a light greyish blue) there is an area of discolouration about $3 \mathrm{~mm}$. wide and with sharply defined edges extending vertically downwards from the central pupillary margin to the base of the iris, and lying in this latter position behind the iris is a small dark patch, wider than the discoloured area, and looking rather like a hyphaema in the wrong place. The " area of discolouration" gives the impression as if the bluish tint had been washed out of the iris, and on closer inspection looks like a patch of atrophic thinning. The pupils are equal, of about normal size, and act normally; the tension of both eyes is equal and apparently normal. She is slightly hypermetropic, and vision with correction $=6 / 9$ in each eye. There are a few striae in each lens : no sign of any other pathological change.

The above is all that is observable as long as she keeps her head up, but when she bends her head there is a very remarkable change. A greyish brown body with minute blood-vessels on the surface has appeared in the front of the iris, extending downwards nearly to the base of the anterior chamber, and of the same width as and lying on the "discoloured area" already described.

- This greyish brown body is rather like heavy lubricating oil pouring over the edge of a cup, but with this peculiarity, that as soon as she raises her head this viscous looking "fluid" gradually rises and slips back over the edges of the iris, and can be seen slowly settling down and resolving itself into the dark substance at the base of the posterior chamber.

It is difficult to describe adequately this most peculiar and striking phenomenon, which can be repeated apparently indefinitely, and 
without any discomfort to the patient, except for the bending of her head, the time taken being a few seconds for the appearance and for the disappearance rather longer.

It is also difficult for me to discuss the case, as I have not previously seen, or heard of, a similar one, but as regards the dimness of sight, of which she mainly complains, I am inclined to think that this is due to the heaping up of the "fluid" as it passes over the concave edge of the iris, and this would naturally be more pronounced when the pupil is contracted in a strong light, and in any case her pupils are naturally getting smaller as she gets older. As regards the aetiology of the condition, I can only suppose that this is a form of the rare kind of cyst described by J. Herbert Parsons as being formed between the retinal layers of the iris, but he does not mention that any of these cysts have the peculiar faculty of appearing in the anterior chamber.

\title{
ACUTE ANTERIOR ETHMOIDITIS IN YOUNG SUBJECTS
}

\author{
BY \\ P. A. HARRY, M.D. \\ OPHTHALMIC SURGEON TO THE ROCHDALE INFIRMARY
}

IN reference to the paper by Mr. Sydney Stephenson on acute anterior ethmoiditis in the August number of the BRITISH JOURNAL OF OPHTHALMOLOGY I may say that I have seen cases such as he describes in children of from 4 to 12 years, but the one I am about to relate is of special interest owing to the age of the patient, namely, 3 weeks :

Ernest P. was sent to the Rochdale Infirmary by Dr. S. B. Brentnall on July 9, 1918. The history was that the left eye had been slightly inflamed since birth. The appearances were those of "a cold in the eye." The condition was treated as an instance of ophthalmia neonatorum for the first ten days.

When I first saw the infant the conditions were as follows: L.E., considerable proptosis and great resistance to pressure backwards. The eye projected directly forwards. Slight inflammation of the conjunctiva accompanied with little discharge. There was a considerable amount of discharge of blood-stained material from the left nostril. The pupillary reactions and the fundus of the left eye were normal. Transillumination through the mouth yielded negative results. A similar remark applies to examination of the nose with Holmes's pharyngoscope. The child was peevish and restless, but there was no rise in temperature and no diarrhoea. 